\title{
Implementation of Pedestrian Navigation System for the Visual Impaired
}

\author{
Young Gun Jang \\ Dept. of Computer \& Information Engineering. Cheongju University, Korea \\ ygjang@cju.ac.kr
}

\begin{abstract}
We implement a portable pedestrian navigation system for the visually impaired. The system consists of a smartphone with a voice recognition function, a budget direction guide and an osteoacusis headset, which can provide auditory guidance directions while recognizing the sound of the surrounding environment. Unlike previous pedestrian navigation smartphone apps, the developed system guides the user in walking direction by the scale of the left and right stereo sound of the headset worn by the user, voice guidance regarding forked or curved paths is given several meters in advance according to the speed of the user, and the user is immediately warned of walking in the opposite direction or proceeding off the path. To overcome GPS positioning errors, we proposed a robust trajectory planning algorithm for position errors. Experimental results show that the average directional angle error is 8.56 degrees (standard deviation: 14.78), average difference between GPS path and scheduled path is $5.55 \mathrm{~m}$ (standard deviation: 3.79 ) and maximum difference is $13.22 \mathrm{~m}$ in the experimental path, based on which can be stated that the user was navigated relatively stable by the developed system.
\end{abstract}

Keywords: Pedestrian navigation, Visually Impaired, Robust trajectory planning

\section{Introduction}

According to a survey [1] of 200 people with visual impairments that was conducted from July 2014 to March 2015 on the need for walking convenience and the development of safety improvement technology, pedestrian navigation is the most essential service for the visually impaired. Of the 52 respondents who answered that they use an activity assistant service, $32(61.5 \%)$ said that they used a pedestrian navigation service including a pedestrian navigation service for commuting, making it the most frequently used service. If a smartphone app were developed that could reliably and conveniently be used for pedestrian navigation, it would be a huge success. According to research of 100 people with visual impairments, while only 63 respondents said they walk a new route about once or twice a week when they walk independently without a guide, $44.8 \%$ of the respondents said that they get lost about once or twice a week. This shows that although it is very uncommon for those with visual impairments to take a new route when walking independently, half frequently experience getting lost. A 2011 study [2] of 9 Americans and 11 Koreans on the needs of the visually impaired for their walking assistance shows that one of the three main demands was to allow them to recover the ability to travel when they get lost.

Devices that assist the visually impaired in walking include electronic travel assistance devices, devices that provide posture or direction information while walking, and devices that provide location information. An electronic travel assistance device is a device that converts and reports information about the surrounding environment to the user. Studies [3-5] have been conducted on the use of such devices to detect and report on dangerous

Received (November 2, 2017), Review Result (January 15, 2018), Accepted (January 22, 2018) 
obstacles, and ultrasound and stereo cameras have been used to detect obstacles. Recently, research on the system supporting walking in indoor spaces [6-8] has been actively conducted, and Yang [6] verified the effectiveness of interface of tactile maps focusing on the tactilegram. Research on mobile pedestrian guidance or information provision systems [9-15] accounts for the largest portion of this research. The study of Kwon [9] classified the information into several dimensions and presented the delivery method in order to convey the spatial information by voice to the visually impaired. Huang [10] proposed an indexing method instead of an exhaustive search method in order to extract contextual information related to a route, and verified its validity. The NAVIG [11] system can obtain information about a route in advance and recognize the travel route, obtain information about the surrounding environment by using the image information obtained from the camera mounted on the head while actually walking, and confirm the position situation of the user. In terms of the estimation of the current position, to fix errors of Global Positioning System (GPS) devices or "shadow" problems, problem solving through camera image and Web DB [16] and a position correction method using an extended Kalman filter [17] were proposed. However, in order to use image information, big data is required, the Kalman filter application further requires sophisticated acceleration and deceleration and gyroscope sensors, and a more powerful computing environment for real-time processing is also needed. Fang et al., [15] proposed an approach based on the needs of pedestrians rather than the existing process or goaloriented approach.

In this study, we implement a mobile system for pedestrian navigation by the visually impaired, and apply it in the real world to verify its usefulness. For mobile implementation, portable devices were adopted. We use the smartphone's built-in GPS sensor to detect the position, and select the smartphone as the basic user interface device. Factors that degrade the accuracy of the GPS location measurement are errors due to structural factors, geometrical errors due to satellite positioning, and errors due to Selective Availability (SA), which is the biggest cause of errors. All of these factors potentially combine to produce an error result, which is called User Equivalent Range Error (UERE). Each error can vary widely depending on time and place. In this study, we propose and implement a robust trajectory planning method for GPS errors in order to prevent a user from being guided in the wrong walking and driving direction due to a GPS error.

\section{Implementation of the System}

Figure 1 shows the configuration of the navigation system for the visually impaired that is implemented in this study. The system consists of a direction guide and an osteoacusis headset that transmits the sounds and voices around a smartphone, and each of them is remotely connected to the open street map (OSM) [18] server and the Mapzen [19] server to obtain the necessary map information and navigation route for a map service and a navigation service. The direction guide is implemented by combining FMT1030 [20], which is an Attitude and Heading Reference System (AHRS), and Ty51822r3 [21], an Arm Mbed-based microprocessor capable of low-power Bluetooth communication. Since electronic maps are used to provide a visual interface, they do not provide services for the visually impaired, but they can be used for communication with nearby people when there is confusion while determining a route. The user runs the smartphone app to access the Mapzen server, receives the node information included in the route, and receives proceeding direction information from the direction guide to combine it with the GPS position information and the route information obtained from the server in order to determine the direction in which the user should walk. The determined direction information is transmitted through the osteoacusis headset. The direction to proceed in is conveyed by controlling the speaker direction and sound intensity of simple 
sounds and outputting it to the two speakers of the headset. The HUI-01 manufactured by Huria was used as the osteoacusis headset.

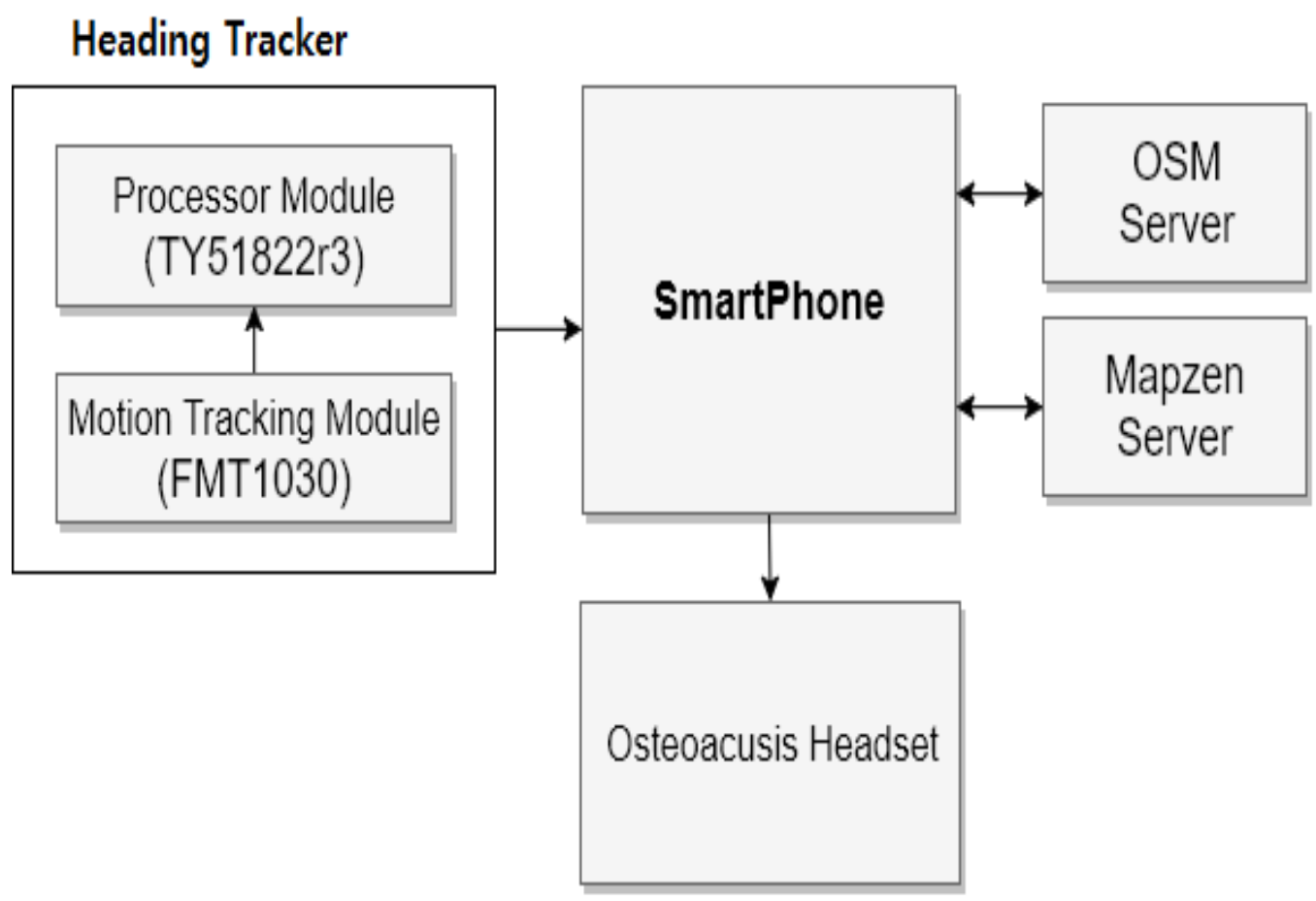

Figure 1. System Configuration

Open Street Map (OSM) was used as the electronic map service. The existing Daum and Naver maps provide an API with limited functions, so the information of the node and link of the map cannot be obtained. As such, it is not possible to input the information of the entrance, convenience facilities and public facilities of the building of Cheongju University to be implemented. In this paper, we used the editing function of OSM to construct by ourselves the data of the campus of Cheongju University based on the satellite map. An example of an electronic map of Cheongju University is shown in Figure 2.

FMT1030, Fairchild 's motion tracking module, was used as the direction sensor. The dynamic precision of direction information is 3 for heading direction, and 3 for roll and pitch, and it has a heading tracking function and a vibration compensation function. Information about angular velocity ( 3 axes), acceleration ( 3 axes), and magnetic field ( 3 axes) are also provided. This sensor has implemented a sensor fusion algorithm called XKF3 based on an extended Kalman filter using 9 axes of angular velocity, acceleration and magnetic field, and has a built-in filter profile that can cope with an environment with a strong magnetic property [20]. It is known that the sensor module is superior to the sensor of $X$ sens in the normal state in terms of AVAR, and the average error angle is 30$70 \%$ smaller [22]. Direction information was acquired from the direction sensor to extract a necessary heading angle, and the function of delivering to the smartphone using Bluetooth low energy (BLE) was implemented using the Ty51822r3 chip made by Switch Science.

The direction guidance system that was implemented is shown in Figure 3. The direction sensor recognizing the actual proceeding direction was connected with Bluetooth, and the smartphone's built-in GPS was used as a position sensor. Direction information can also be used effectively for position estimation [23]. The position 
information was acquired every $1 \mathrm{~Hz}$, and the direction information was acquired every 5 Hz to use.

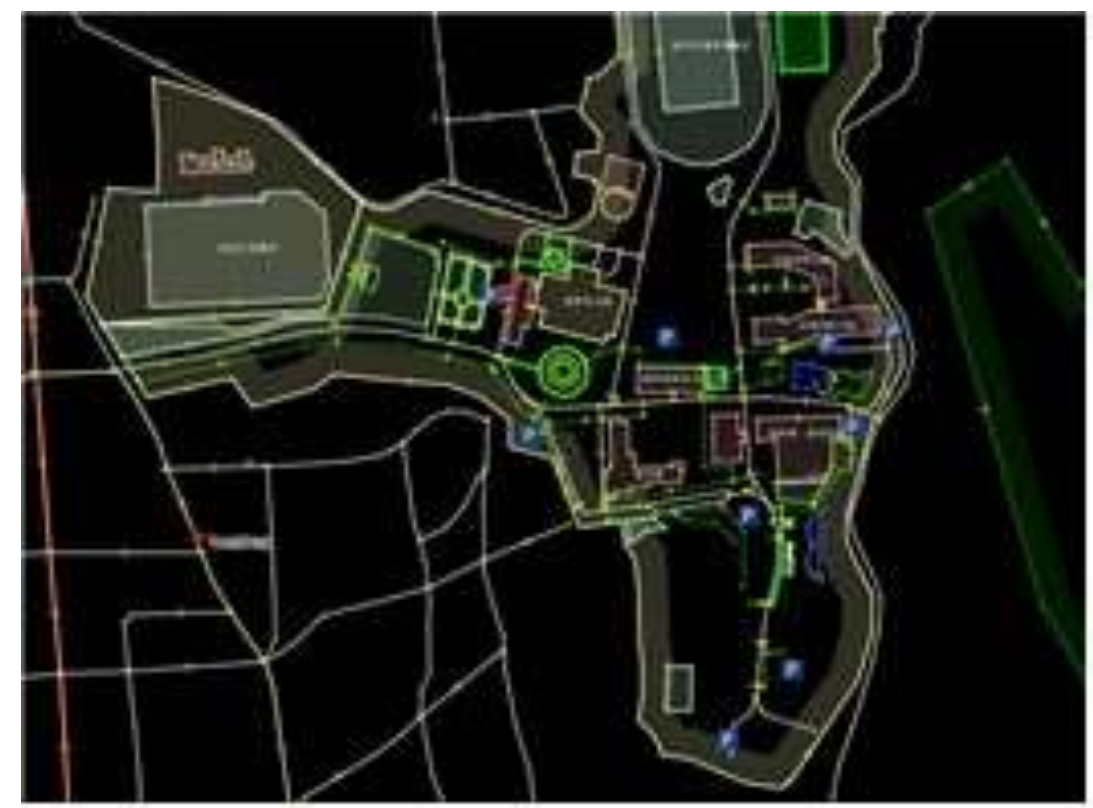

\section{Figure 2. A Construction Example of OSM Map in Cheongju University}

The position information acquired from the GPS sensor is actually inaccurate and may be influenced by the surrounding environment on the route, thereby increasing the error. Some papers using GPS information have reported errors of up to 17 meters [17]. Generally, navigation consists of nodes and detailed links [24]. The entire route can be said to be a set of such nodes and detailed links. Two nodes and one detailed link connecting them constitute one segment. In this study, we used the Mapzen map service platform to obtain the overall nodes and detailed links. Figure 4 shows the concept of nodes and detailed links [9]. The trajectory planning in one section calculates the position closest to the planned route from the position information obtained from the GPS at regular time intervals and estimates it as its own position information. Then, it compares the position to determine whether or not the position is the same as the decision point, and if it's not the same the user continues to walk.

It is assumed that if users feel that they are walking in the right direction, it's consistent with the fact that they think the location information obtained from the GPS is reliable up to the route that has been taken. This view assumes that the routes that have been taken by the pedestrians are on the psychological safety layer, which was proposed by Huang et al. The problem when the position information that does not match the movement direction and speed acquired from the sensor in the confidence interval are input from the GPS is how to estimate the current position. One is an estimation method based on dead reckoning, and the other is an estimation of the current position assuming that the user is walking in the right direction. This study chose the latter method. In this paper, we chose a heuristic method instead of using the method of reducing the error of GPS position information, such as the application of an extended Kalman filter as a method for estimating the current position. In other words, the coordinates intersecting the normal perpendicular to the planned route at the position acquired from the GPS were regarded as the current position. This method works effectively when the direction guidance is conducted normally and the deviation from the planned route is within a certain distance. The direction guide was implemented by using AHRS with an extended Kalman filter. If it is judged that the user has deviated by a certain distance, the route is automatically set 
again using the Mapzen server.

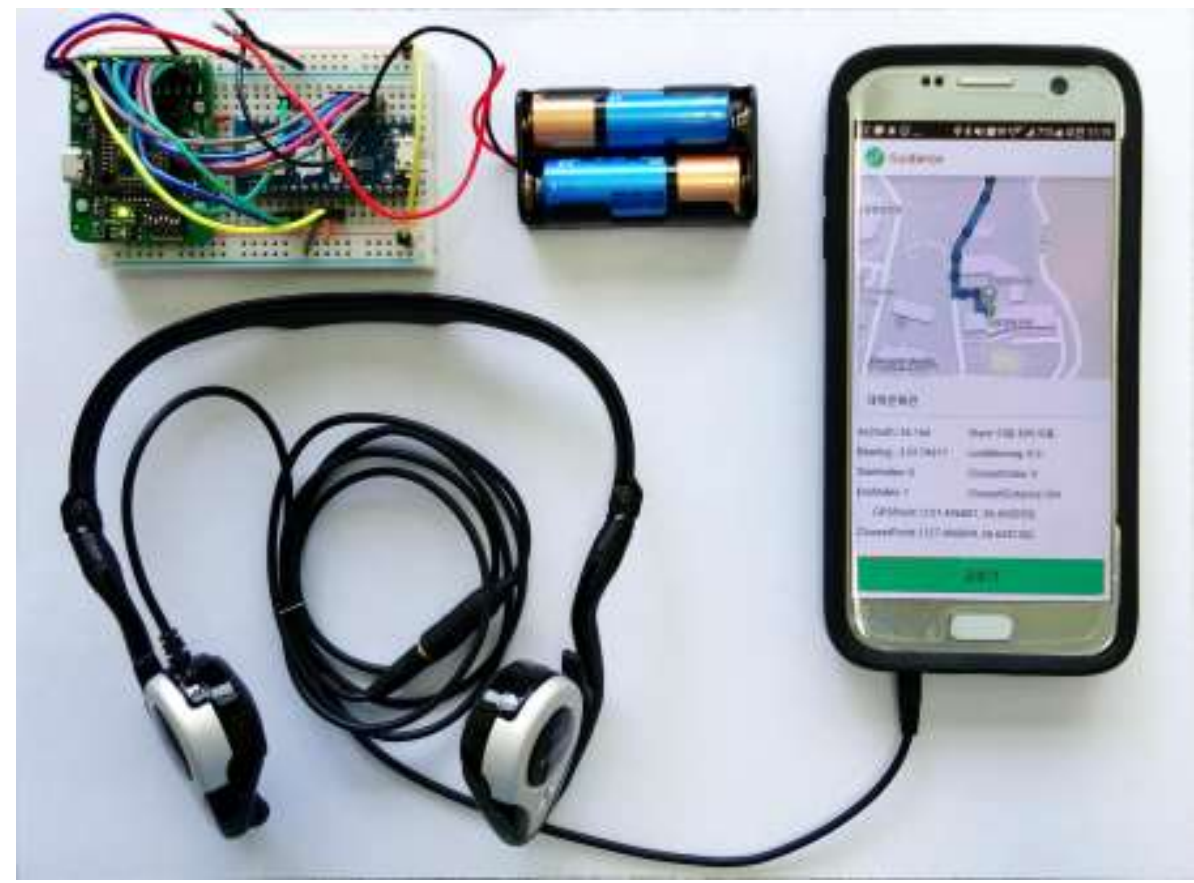

Figure 3. Implemented Pedestrian Navigation System

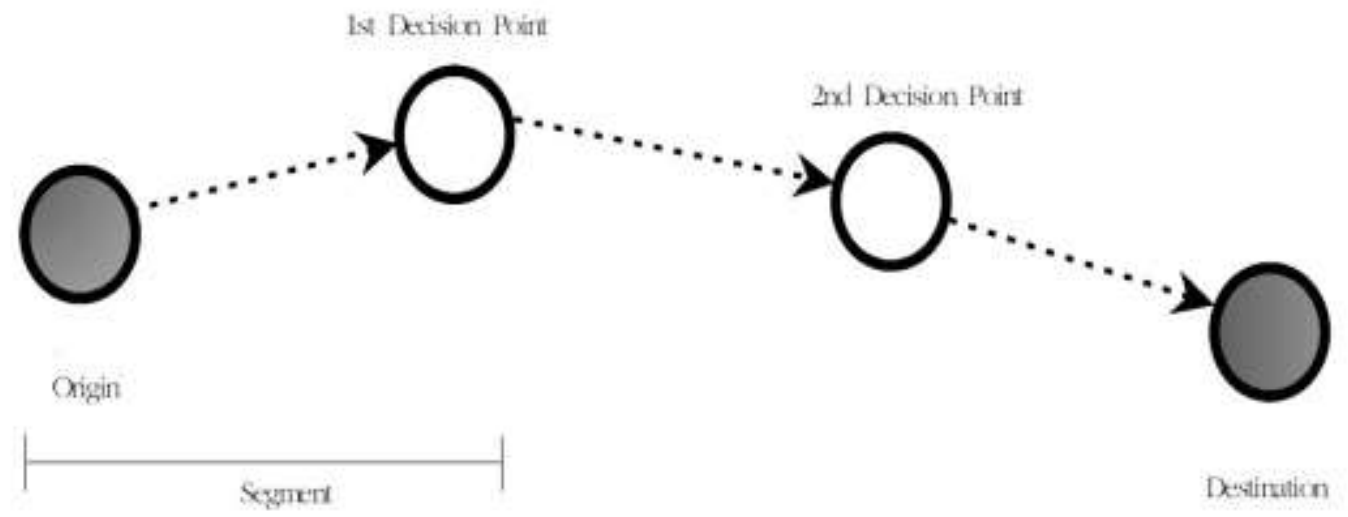

Figure 4. Configuration Elements of Navigation

We calculated the bearing angle to proceed from the node information acquired from the Mapzen server, and compared it to the actual heading angle received from the direction sensor. The difference was controlled by converting the beep sound intensity in the left and right speakers of the osteoacusis headset. The representation of the difference of the angles by the sound intensity was determined by the experimental value. The navigation route is expressed as a sequential set of nodes generated between the current location and the destination. In terms of node arrival during the movement between the nodes, if the difference between latitude and longitude values is within a thousandth, the node is determined to arrive, and the difference is within $7 \mathrm{~m}$. The bearing angle to proceed with is calculated using latitude and longitude between the next nodes at the current node. Figure 5 shows the flow of information between devices in the implemented system. 


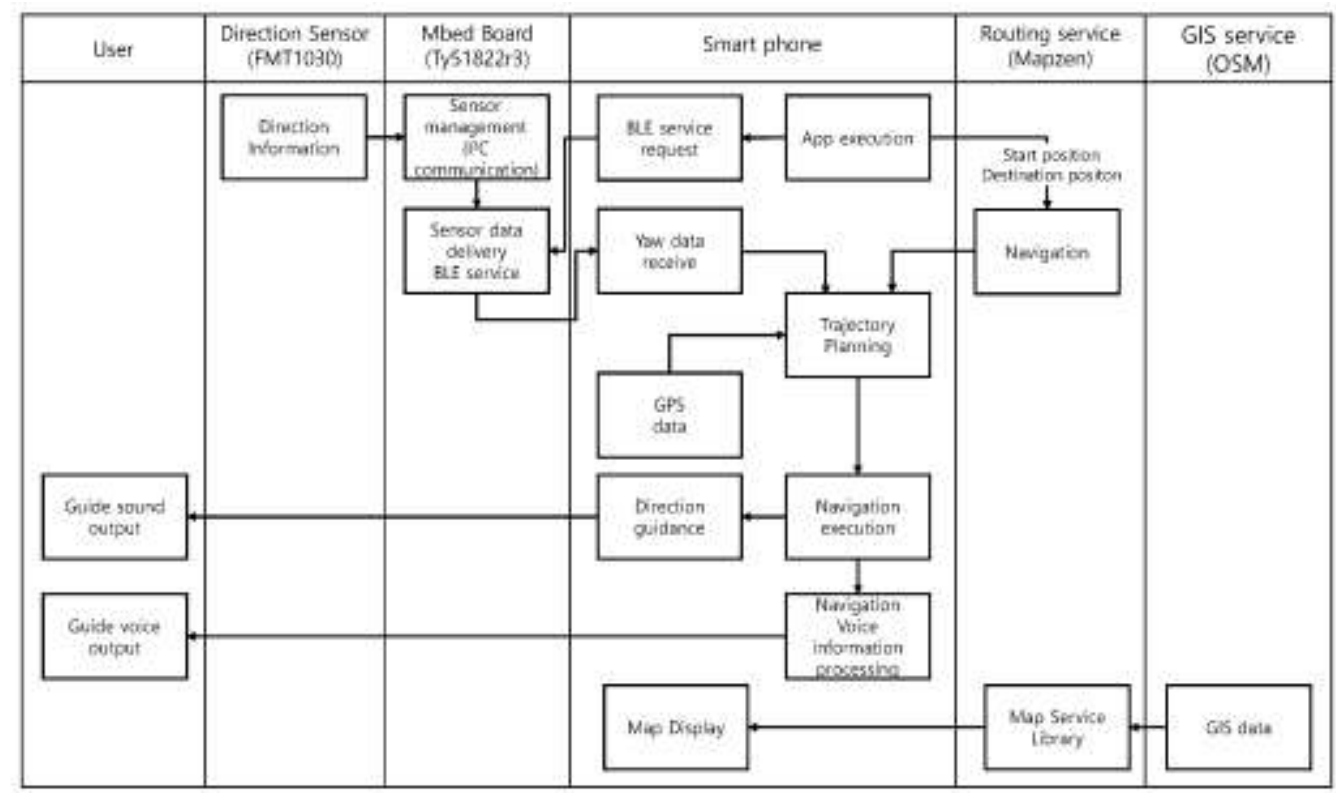

Figure 5. Information Flow between Devices in the System

\section{Experiments and Evaluation}

To evaluate the function and performance of the navigation during walking, this study implemented an evaluation tool which records walking routes and direction information on smartphones and displays the file on a computer as a map. Figure 6 shows a screen displaying the mapping process in the evaluation tool that was implemented. A user can select the recorded data file by pressing the data confirmation button, and see the planned route indicated as dotted lines and the actual route indicated as lines with alternating long and short dashes by pressing the walking record map button. The user can download the file from a smartphone and use it for analysis for more information.

In the experiment, a person without disabilities wore an eye patch and walked with the guidance of the implemented walking guide. Figure 7 shows the experimental screen. For the experiment, we designated the starting and returning locations on the university campus, specified a route to return to the starting location, and included both gently curved paths and suddenly curved paths between the two points. Evaluation tools were used to evaluate the appropriateness of the induced walking. Figure 8 shows the proceeding route that was displayed on the GIS shown as a result of this experiment. The planned route, the actual route, and the proceeding direction are shown on the right side of the figure by enlarging a part of the proceeding route. The black dotted line is the line connecting the nodes formed by Mapzen, and is the planned route. The alternating long and short dashes forming a line connect the locations measured by the GPS. The full line indicates the proceeding direction detected by $2 \mathrm{~Hz}$ unit on the proceeding route. As shown in the picture, the user arrived at the destination from the starting point by using only the sound guide. Although some differences from the planned routes are shown on some routes, this is largely due to the GPS error, and the actual proceeding route showed a smaller difference from the planned route shown in the figure. Figure 9 shows the planned bearing angle and the measured heading angle of the walking route in this experiment. The average of the difference between the two angles is 8.56 and the standard deviation is 14.78. This shows that the user followed the directions relatively well, and could reach the destination precisely. The difference between the GPS measurement location and the location closest to the planned route was $13.22 \mathrm{~m}$ at maximum, the average was $5.55 \mathrm{~m}$, and the standard deviation was 3.79. It is believed that after a user becomes accustomed to the use of the auditory guide, better results can be achieved. Using the apps implemented 
in this paper greatly reduces the chance of getting lost compared to using a smartphone app such as Kakao map without a direction guide. Indeed, Kakao map visually displays the location of the user on the smartphone only after the user changes the direction and proceeds several tens of meters.

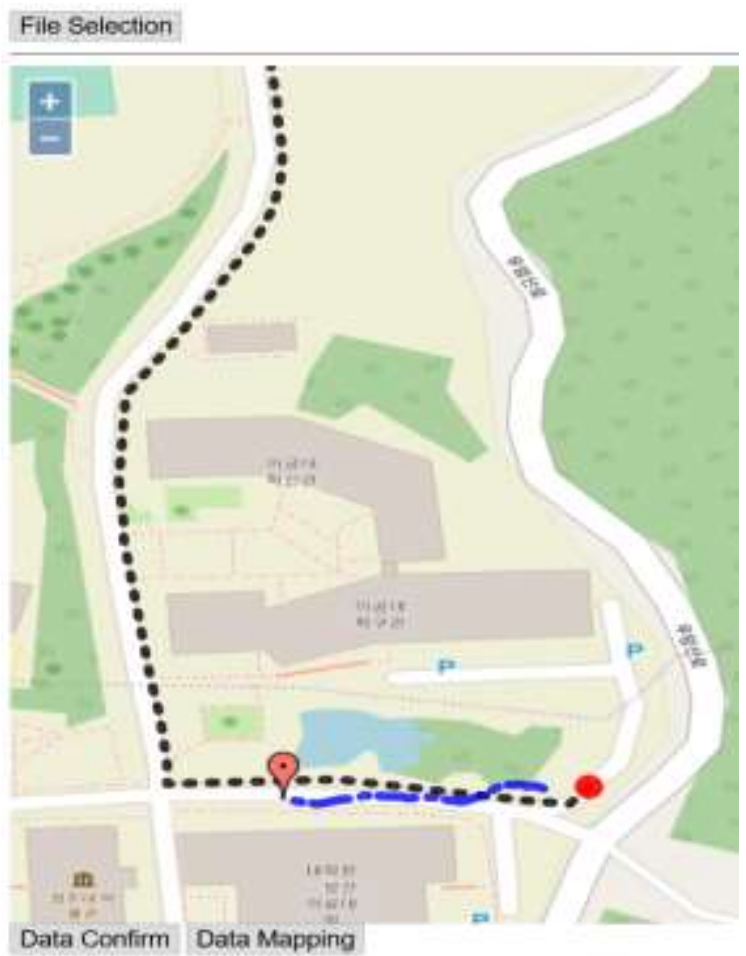

Figure 6. Mapping Process for Pedestrian Record

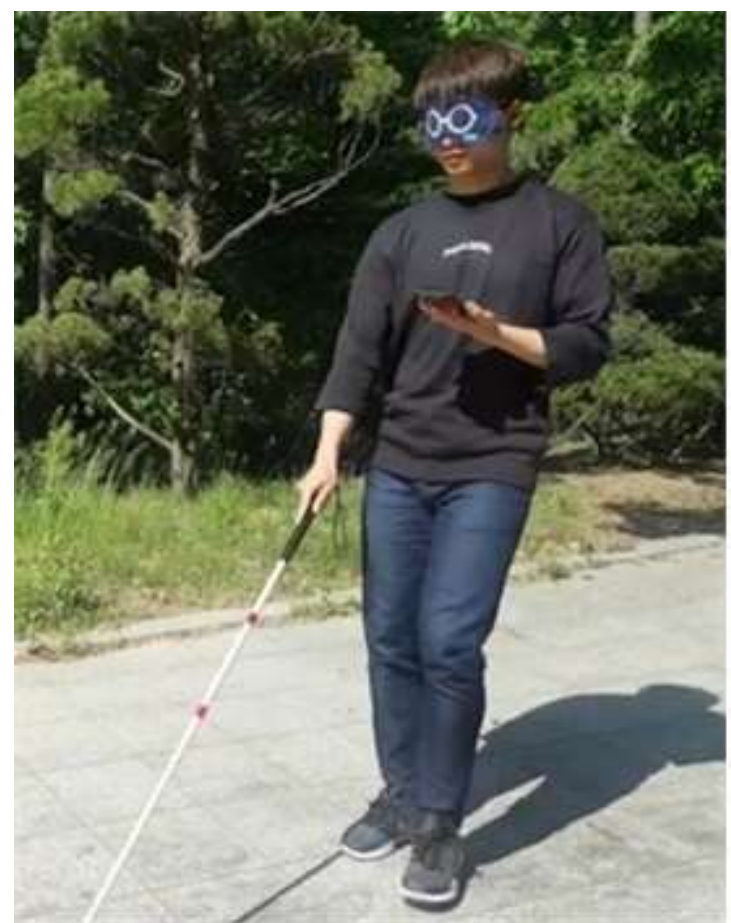

Figure 7. An Experimental Scene 


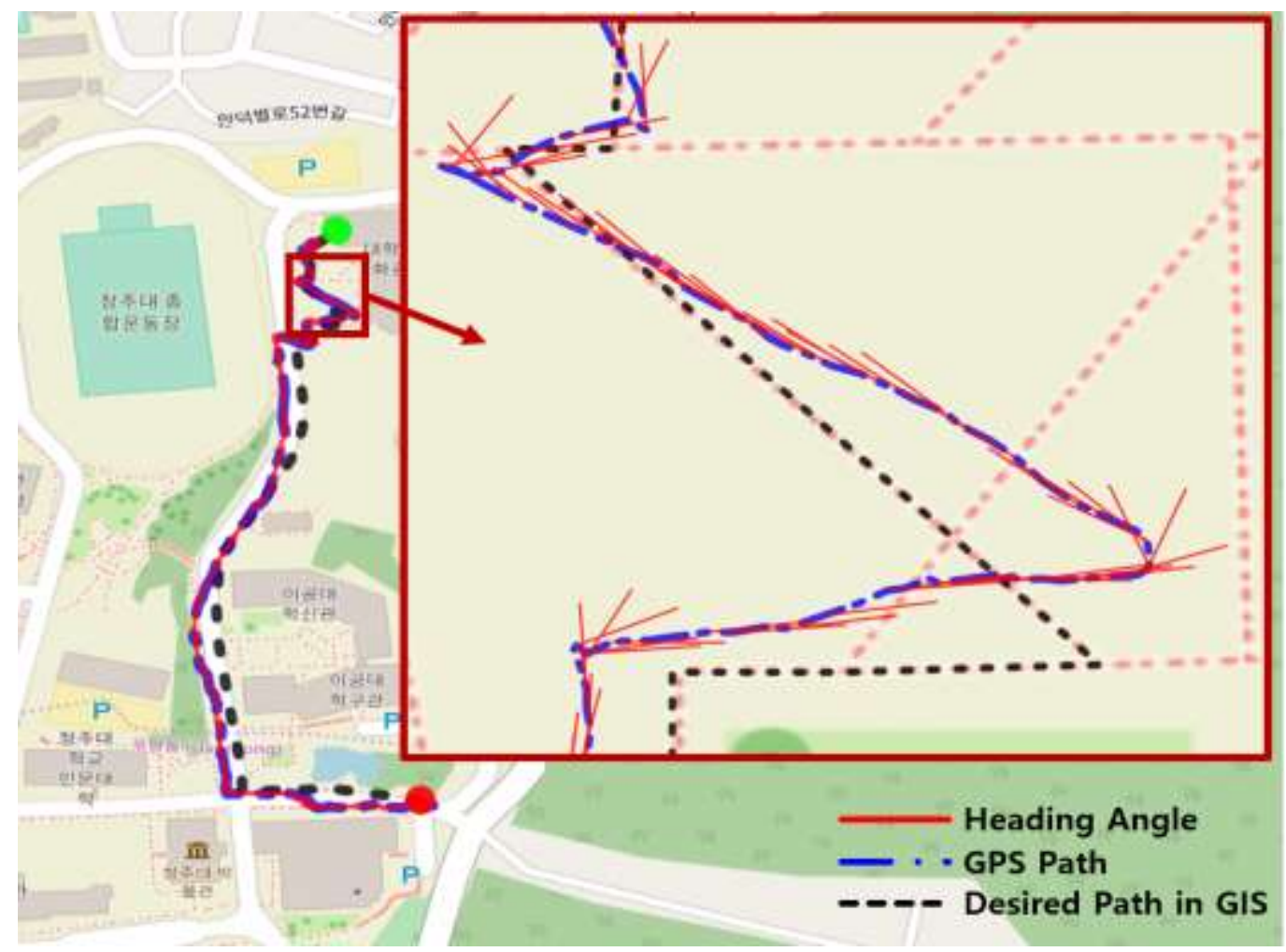

Figure 8. Experimental Results of Scheduled Path, GPS Path and Pose Direction

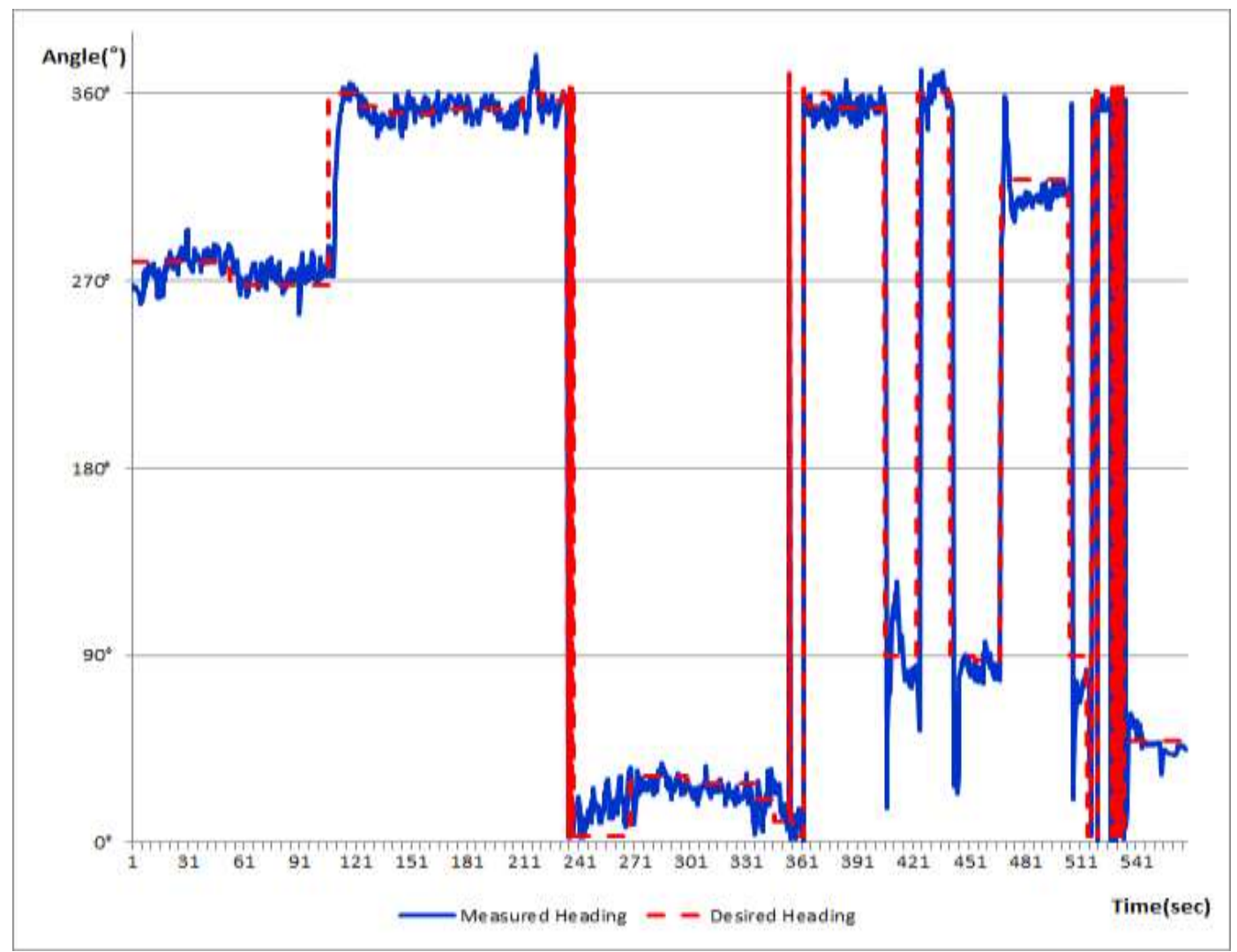

Figure 9. Time based Progress of Scheduled Bearing Angle and Measured Heading Angle 


\section{Conclusion}

We implemented a portable pedestrian guidance system for the visually impaired using a high precision direction sensor, an osteoacusis headset and a smartphone. Through testing the implemented system on the campus of Cheongju University, it was confirmed that the user had no difficulties in navigation with the sound guidance using the osteoacusis headset, and the problem of walking route estimation caused by GPS error can be solved by the proposed heuristic algorithm. To solve the problem of not finding the current position that may occur when passing through GPS 'shadow' areas, we plan to supplement the system by further developing the position estimation algorithm.

\section{Acknowledgments}

This paper is a revised and expanded version of a paper entitled "Pedestrian Navigation System Using Low Cost AHRS for the Visually Impaired" presented at the $14^{\text {th }} 2017$ International Interdisciplinary Workshop Series, Daejeon, Dec. 2017.

\section{References}

[1] Electronics and Telecommunications Research Institute, Korea Blind Union, A Study of Requirements for Pedestrian Convenience of Visually impaired, Final report, (2015) March 27.

[2] P.-A. Quiñones, "Supporting Visually Impaired Navigation: A Needs-finding Study”, CHI 2011, (2011), May 7-12.

[3] L. Kim, "Improvement of an Electronic Aid for the Blind using Ultrasonic and Acceleration Sensors", Journal of KISS: Software and Applications, vol. 36, no. 4, (2009), pp. 291-297.

[4] A. Rodr'1guez, "Assisting the Visually Impaired: Obstacle Detection and Warning System by Acoustic Feedback", Sensors, vol. 12, (2012), pp. 17476-17496.

[5] D. Dakopoulos and N. G. Bourbakis, "Wearable Obstacle Avoidance Electronic Travel Aids for Blind: A Survey", IEEE Transactions on Systems, Man and Cybernetics- Part C: Applications and Reviews, vol. 40, no. 1, (2010) January, pp. 25-35.

[6] Y. Seung Ho, "A Study on the Walking Aids'Interface for Independent Indoor Walking of the Visually Impaired", Kookmin University, Phd thesis, (2012).

[7] D. Jain, "Path-Guided Indoor Navigation for the Visually Impaired Using Minimal Building Retrofitting", ASSETS'14, (2014) October 20-22.

[8] A. Ganz, "PERCEPT Indoor Navigation System for the Blind and Visually Impaired: Architecture and Experimentation", International Journal of Telemedicine and Applications, vol. 2012, Article ID 894869.

[9] K.-S. Kwon, "Spatial Information and Providing Methods in Mobile Pedestrian Navigation for the Visually Impaired", Journal of Korean Cartographic Association, vol. 13, no. 2, pp. 95-111, (2013).

[10] B. Huang and N. Liu, "Mobile Navigation Guide for the Visually Disabled", Transportation Research Record, No. 1885, (2004), pp. 28-34.

[11] B. F. G. Katz, "NAVIG: Guidance system for the visually impaired using virtual augmented reality", Technology and Disability, vol. 24, (2012), pp. 1-16.

[12] K. Ramarethinam, "Navigation System for Blind People Using GPS \& GSM Techniques", IJAREEIE, vol. 3, no. 2, (2014), pp. 398-405.

[13] A. J. May, "Pedestrian navigation aids: information requirements and design implications", Pervasive Ubiquitous Computing, vol. 7, (2003), pp. 331-338.

[14] M. Minock, "Prediction and Scheduling in Navigation Systems", GeoHCI Workshop at CHI 2013, Paris, France, (2013) April 27-28.

[15] Z. Fang, "What about people in pedestrian navigation?", Geo-spatial Information Science, vol. 18, no. 4, (2015), pp. 135-150.

[16] K.-h. Kim and S.-W. Lee, "Positioning System for the Blind Navigation", Journal of Korean Institute of Next Generation Computing, vol. 8, no. 4, (2012) August, pp. 6-16.

[17] J.-k. Yoo, "Development of Localization Tracking System and User Interface of Guiding Robot for the Visually Impaired", Journal of Korea Information Processing Society D, vol. 12, no. 3, (2005), pp. 481491.

[18] Open street map, https://www.openstreetmap.org/\#map=7/35.948/127.736.

[19] Get driving, biking, walking and transit navigation in your app, https://mapzen.com/.

[20] Fairchild Semiconductor, FMT 1000 series Motion Tracking Module with Output of Orientation, Inertial Motion Data and Magnetic Field. Fairchild Semiconductor; South Portland, ME, USA, (2016).

[21] Switch Science mbed TY51822r3, https://developer.mbed.org/platforms/Switch-Science-mbedTY51822r3/. 
International Journal of Grid and Distributed Computing

Vol. 11, No. 2 (2018)

[22] A. Szczesna and P. Skurowski, "Inertial Motion Capture Costume Design Study", Sensors, vol. 17, no. 3, (2017) March, pp. 612-631.

[23] K.-j. Kim, "Dead-Reckning Error Correction Using Orientation Information”, 2007 Proceeding of KIIT Summer Conference, (2007), pp. 238-243.

[24] M. Raubal and S. Winter, 2002 Enriching Wayfinding Instructions with Local Landmarks, Lecture Notes in Computer Science, 2478, pp. 243-259. 